\title{
Complement as a target in COVID-19?
}

Antonio M. Risitano ${ }^{1}$, Dimitrios C. Mastellos $\circledast^{2}$, Markus Huber-Lang ${ }^{3}$,
Despina Yancopoulou ${ }^{4}$, Cecilia Garlanda ${ }^{5,6}$, Fabio Ciceri ${ }^{7}$ and John D. Lambris $\circledast^{8 凶}$

There is an urgent need to develop effective therapies for COVID-19. Here, we urge immunologists and clinicians to consider the potential of targeting the complement system in these patients.
'Department of Clinical Medicine and Surgery, Federico II University of Naples, Naples, Italy.

${ }^{2}$ National Center for Scientific Research 'Demokritos', Aghia Paraskevi, Athens, Greece.

IInstitute of Experimental Trauma-Immunology, University Hospital of UIm, Ulm, Germany.

${ }^{4}$ Amyndas Pharmaceuticals, Glyfada, Greece.

${ }^{5}$ Humanitas Clinical and Research Center, IRCCS, Rozzano, Milan, Italy.

${ }^{6}$ Humanitas University, Pieve Emanuele, Milan, Italy.

'IRCCS Ospedale San Raffaele, University Vita-Salute San Raffaele, Milan, Italy.

${ }^{8}$ Department of Pathology and Laboratory Medicine, Perelman School of Medicine, University of Pennsylvania, Philadelphia, PA, USA.

凶e-mail: ciceri.fabio@hsr.it; lambris@pennmedicine. upenn.edu

https://doi.org/10.1038 \$41577-020-0320-7
Most patients who become critically ill following infection with SARS-CoV-2, the causative agent of COVID-19, develop acute respiratory distress syndrome $(\mathrm{ARDS})^{1}$. The deterioration of lung function has been attributed to a maladaptive immune response rather than increased viral loads. One theory is that the activation of lung-resident immune cells via pattern-recognition receptors drives the release of pro-inflammatory cytokines and extravasation of blood neutrophils and monocytes into the bronchi. These cells may disrupt the air-blood barrier by causing collateral tissue damage, particularly to airway epithelial cells and vascular endothelial cells, which express the ACE2 entry receptor for SARS-CoV-2; the damage of vascular endothelial cells may account for thrombotic microangiopathies. Currently, COVID-19 management is limited to symptomatic and palliative treatment. Efforts are underway to develop more effective therapies, with trials including various antiviral drugs, anti-inflammatory agents and the anti-malarial drug hydroxychloroquine. Ongoing clinical trials of IL-6 and IL- 1 blockade in patients had promising preliminary results and may build a basis for combinatorial immunotherapy approaches.

Complement is an integral component of the innate immune response to viruses and an instigator of proinflammatory responses ${ }^{2,3}$. A recent study of SARS-CoV, which is closely related to SARS-CoV-2, found that activation of complement component $\mathrm{C} 3$ exacerbates disease in SARS-CoV-associated ARDS ${ }^{4}$. C3-deficient mice infected with SARS-CoV exhibited less respiratory dysfunction, despite equivalent viral loads in the lungs, and this was associated with decreased lung infiltration of neutrophils and inflammatory monocytes and lower levels of cytokines and chemokines in both the lungs and sera $^{4}$. This suggests that $\mathrm{C} 3$ inhibition may also alleviate the inflammatory lung complications of SARS-CoV-2 infection. The prominent decrease in lung-infiltrating neutrophils and the reduced levels of both intrapulmonary and plasma IL-6 seen in SARS-CoV-infected C3-deficient mice suggests the potential of combining C3 inhibitors with anti-IL-6 regimens. Furthermore, the upstream positioning of $\mathrm{C} 3$ signalling in the innate immune cascade further argues for the broader antiinflammatory potential of $\mathrm{C} 3$ blockade with agents such as AMY-101 (REF. ${ }^{3}$ ), which is currently being tested in patients with COVID-19. C3 inhibition could simultaneously block C3a and C5a generation, as well as intrapulmonary C3 activation and IL-6 release from alveolar macrophages, or other cells that express C3a receptors (C3aRs) and/or C5a receptors (C5aRs), thereby ameliorating lung injury. Ex vivo whole blood infection models with the compstatin C3 inhibitor AMY-101 have shown that this interferes with IL-6 release ${ }^{3}$.

An important caveat is that, although complement activation has been associated with the pathophysiology of ARDS caused by various underlying diseases ${ }^{5}$, clinical data on the role of complement activation in the development of SARS-CoV-2-associated ARDS are scarce. A recent preprint study reported that lung biopsy samples from patients with severe COVID-19 showed widespread complement activation, characterized by C3a generation and C3-fragment deposition ${ }^{6}$. A prominent increase of serum C5a levels was also observed. Importantly, treatment of patients with an anti-C5a antibody led to immediate clinical improvement, as measured by increased lung oxygenation and decreased systemic inflammation ${ }^{6}$. C5 inhibitors have been safely used in the clinic for almost 15 years, and their use in initial clinical trials is supported by the well-established role of the C5a-C5aR axis in the pathophysiology of ARDS. However, C5 inhibition by these agents can be partial, allowing residual terminal pathway activity to skew efficacy in cases of excessive complement activation, which is often seen in infections. Also, these drugs will not affect the C3a-C3aR axis. Proximal complement inhibitors (which target C3 or its upstream activators) could be more effective, but these are still in clinical development, and none has yet been approved, although limited data from phase II clinical trials are available. Given the organization of the complement system, all these agents could potentially prevent the initial steps leading to overt lung inflammation (FIG. 1). A broader inhibitory effect on the maladaptive inflammatory response may be achieved with anti-C3 agents (such as AMY-101), and some pathway-specific upstream inhibitors may also show efficacy (for example, lectin pathway inhibitors). The benefits of specifically inhibiting either the alternative pathway or classical pathway remain to be clarified. 


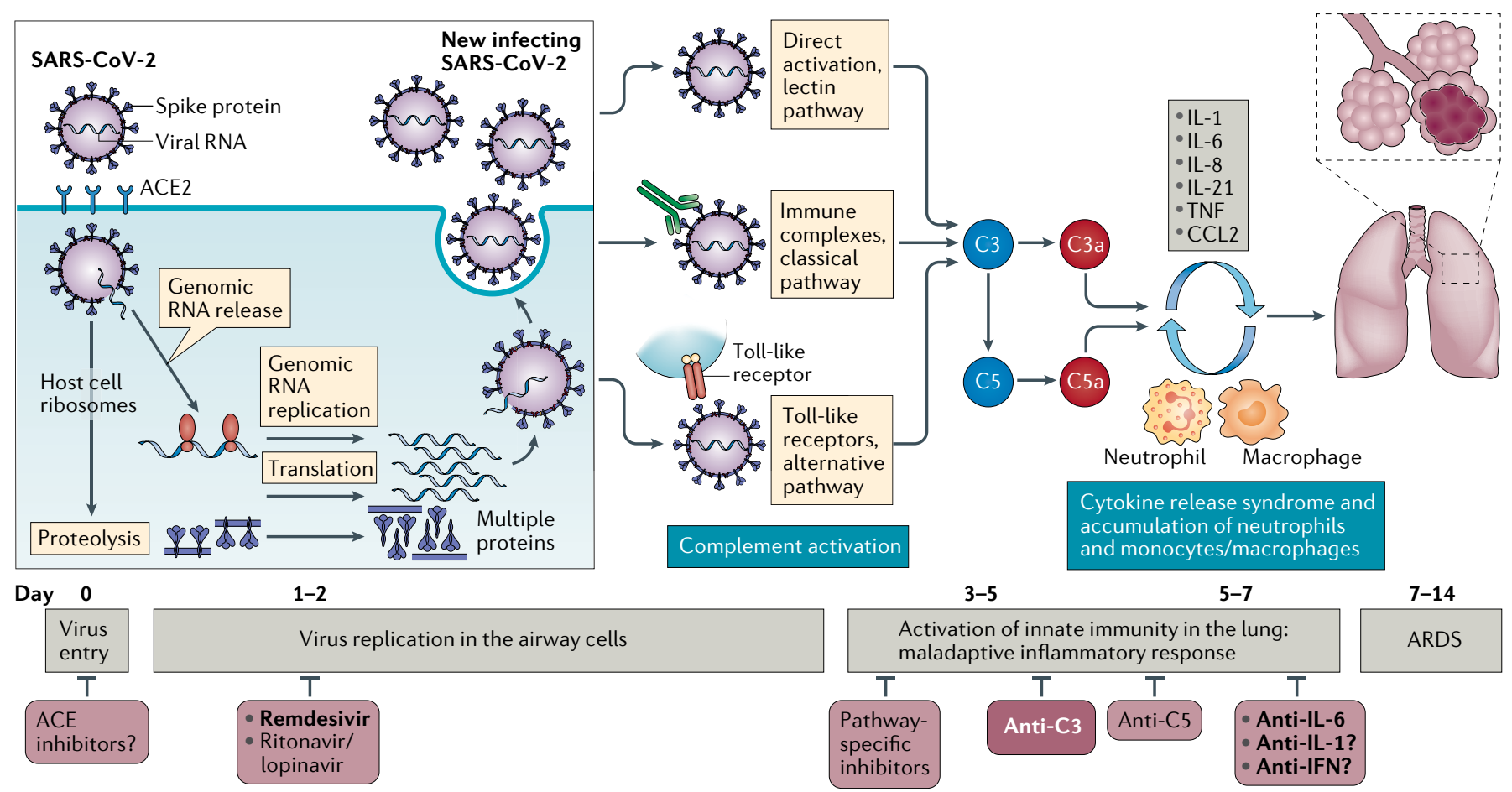

Fig. 1 | Targeting complement in SARS-CoV-2-associated lung injury. Complement activation may contribute to the maladaptive inflammatory response seen in some patients with severe COVID-19. Inhibition of C 3 or C5 may have therapeutic potential. ARDS, acute respiratory distress syndrome.

Additional questions remain concerning the therapeutic use of complement inhibitors for COVID-19. Only a small proportion of patients develop aggressive disease but reliable clinical indicators to identify these patients early in disease progression are lacking. The time window for optimal intervention and the patient populations that could benefit from therapeutic complement inhibition have yet to be established. Biomarkers of complement activity are not routinely used, as most of them are unstable and have a very short half-life. However, the combination of clinical indicators of ARDS progression with known biomarkers of inflammation (C-reactive protein, plasma IL-6 levels and ferritin) would allow identification of patients that could benefit from complement inhibition. In these advanced stages of COVID-19, C3 inhibition has the potential to broadly control not only ARDS but also the systemic inflammation affecting the microvascular beds of the kidney, brain and other vital organs, which seems to be a complication in severe cases.

Complement is a key player of protective immunity against pathogens, but its excessive or deregulated activation may result in collateral tissue injury. However, complement inhibitors are currently only used in rare human diseases, such as paroxysmal nocturnal haemoglobinuria. In these unprecedented times, we would encourage all complement-dedicated pharmaceutical companies, as well as individual scientists, to actively contribute to our efforts to understand the role of complement in COVID-19.

1. Guan, W.-j et al. Clinical characteristics of coronavirus disease 2019 in China. N. Engl. J. Med. https://doi.org/10.1056/NEJMoa2002032 (2020).

2. Li, G. et al. Coronavirus infections and immune responses. J. Med. Virol. 92, 424-432 (2020).

3. Mastellos, D. C., Ricklin, D. \& Lambris, J. D. Clinical promise of next-generation complement therapeutics. Nat. Rev. Drug Discov. 18, 707-729 (2018)

4. Gralinski, L. E. et al. Complement activation contributes to severe acute respiratory syndrome coronavirus pathogenesis. $\mathrm{mBio}$ https://doi.org/10.1128/mBio.01753-18 (2018).

5. Zilow, G. et al. Generation of anaphylatoxin C3a in plasma and bronchoalveolar lavage fluid in trauma patients at risk for the adult respiratory distress syndrome. Crit. Care Med. 20, 468-473 (1992).

6. Gao, T. et al. Highly pathogenic coronavirus $\mathrm{N}$ protein aggravates lung injury by MASP-2-mediated complement over-activation. medRxiv Preprint at https://doi.org/10.1101/2020.03.29.20041962 (2020).

Author contributions

The authors contributed equally to all aspects of the article.

\section{Competing interests}

J.D.L. is the founder of Amyndas Pharmaceuticals and inventor of patents related to complement inhibitors, including a technology licensed to Apellis Pharmaceuticals. A.M.R. has received support from or served as an adviser for Biocryst, Achillion, Alexion, Alnylam, Amyndas, Apellis, Novartis, Roche, Omeros, Pfizer, Rapharma, Samsung and Sanofi. M.H.-L. holds a patent relating to $\mathrm{C} 5 \mathrm{a}$ inhibition licensed to InflaRx. D.Y. is the Managing Director of Amyndas Pharmaceuticals. D.C.M., C.G. and F.C. declare no competing interests. 